\title{
AVALIAÇÃO QUALITATIVA DAS ESPÉCIES ARBÓREAS DO PARQUE ANTÔNIO PIRES DE CAMPOS EM CUIABÁ-MT
}

\author{
Gustavo Henrique da Guia ${ }^{1}$, Joana Maria Ferreira Albretch ${ }^{2}$, Thelma Shirlen Soares ${ }^{3}$, \\ Miranda Titon ${ }^{3}$
}

(recebido em 23.03.2008 e aceito para publicação em 25.09.2008)

\section{RESUMO}

Este estudo teve por objetivo realizar a avaliação qualitativa da arborização do Parque Antônio Pires de Campos em Cuiabá-MT. Realizou-se o levantamento identificando as espécies em toda a extensão do Parque e analisando os parâmetros altura, qualidade da copa, caule e raiz, presença de pragas e danos físicos. Foram amostrados 1502 indivíduos, representando 50 espécies e 26 famílias. Verificou-se que o Parque é composto principalmente por espécies nativas (92\%) sendo que as espécies em maior número de indivíduos são: gonçaleiro (Astronium fraxinifolium), farinheira (Albizia hasslerii) e o louro (Cordia glabrata). Em relação ao estado fitossanitário, $61,26 \%$ do total das árvores apresentaram-se como íntegras, ou seja, possuíam características típicas da espécie e em pleno vigor de desenvolvimento.

Palavras-chave: Árvores urbanas; arborização de parques.

\begin{abstract}
The study is designed to evaluation the qualitative arborization of the Parque Antônio Pires de Campos in Cuiabá-MT. The survey was become identifying the species in all the extension of the Park and analyzing the parameters height, quality of the crown, stem and root, physical damages and presence of pest. The survey totaled 1502 individuals, 50 species belonging to 26 families. The Park is composed mainly for native species (92\%), the species with the highest number of individuals were: gonçaleiro (Astronium fraxinifolium), farinheira (Albizia hasslerii) and the parrot (Cordia glabrata). $61.26 \%$ of the total of the trees had been presented as health, or either, they are characteristic typical of the species and in vigor of development.
\end{abstract}

Keywords: Urban trees; urban forest in parks.

\footnotetext{
${ }^{1}$ Engenheiro Florestal graduado pela Universidade Federal de Mato Grosso.

${ }^{2}$ Professora da Faculdade de Engenharia Florestal da Universidade Federal de Mato Grosso, Av.

Fernando Correa da Costa, s/n, Cep: 78.900-000, Cuiabá-MT. rsam@ufmt.br

3 Professora do Departamento de Engenharia Florestal da Universidade Federal dos Vales do Jequitinhonha e Mucuri, Rua da Glória, 187, Centro, Cep: 39.100-000, Diamantina-MG. thelma.soares@ufvjm.edu.br; titonmiranda@yahoo.com.br
} 


\section{INTRODUÇÃO}

O ambiente urbano, desde a revolução industrial, vem sofrendo alterações que modificaram a harmonia entre os elementos naturais. Atualmente, os efeitos das ações antrópicas nas áreas urbanas repercutem de forma qualitativa e quantitativa com a fragilização das áreas verdes e a diminuição da percentagem de cobertura vegetal urbana, provocando ilhas de calor e alterando o clima local (ALBRECHT, 1998).

A arborização das cidades constitui-se em um elemento de grande importância para a elevação da qualidade de vida da população, seja em grandes centros urbanos quanto em pequenas cidades. Com suas características, são capazes de controlar muitos efeitos adversos do ambiente urbano, contribuindo para uma significativa melhoria na qualidade de vida, pois melhoram o ambiente urbano tanto no aspecto ecológico quanto na sua estética (MENESES et al., 2003).

A vegetação urbana contribui para harmonia da paisagem quebrando a dureza e a rigidez do concreto, criando linhas mais suaves e naturais (PAIVA e GONÇALVES, 2002).

O Parque Antônio Pires de Campos, mais conhecido como Morro da Luz representa o marco inicial do surgimento do município de Cuiabá em Mato Grosso. O Parque é privilegiado por possuir ao mesmo tempo característica de área verde central, respiradouro das ilhas de calor, e de fazer parte do conjunto arquitetônico-urbanístico e paisagístico do município, tornando-se assim, de interesse ecológico, cientifico, turístico histórico, cultural e patrimonial.

Neste contexto, estudos que visem avaliar a situação atual da flora do parque apresentam-se como subsídios para realizar análises para seu replanejamento e sua reestruturação.

Este estudo teve como objetivo a avaliação qualitativa da composição vertical do Parque Antônio Pires de Campos, visando identificar, classificar e apresentar uma listagem das espécies arbóreas encontradas com suas respectivas famílias e número de ocorrência, além de avaliar o estado fitossanitário das árvores.

\section{MATERIAL E MÉTODOS}

O Parque Antônio Pires de Campos conhecido popularmente como Morro da Luz, situa-se no bairro Bandeirantes, região central de Cuiabá-MT, na latitude $15^{\circ} 35^{\prime} 56^{\prime \prime} \mathrm{S}$ e longitude $56^{\circ} 06^{\prime} 01^{\prime \prime} \mathrm{W}$ com altitude média de $165 \mathrm{~m}$. Possui área de aproximadamente 6 ha e é considerado, de acordo com o Decreto Municipal 870/83, patrimônio histórico, paisagístico e ecológico.

Foram medidos todos os indivíduos arbóreos com cap (circunferência a 1,30 m do solo) maior ou igual a $15,0 \mathrm{~cm}$. A identificação taxonômica das espécies avaliadas foi efetuada mediante consultas a herbários, consultas a especialistas e por meio de literatura 
especializada. A sinonímia e a grafia dos taxa foram atualizadas mediante consulta ao índice de espécies do Royal Botanic Garden e do banco de dados do Missouri Botanical Garden, disponível na página http://mobot.mobot.org/W3T/Search/vast.html. O sistema de classificação adotado foi o APG II (2003). O material botânico coletado em estado reprodutivo está depositado no Herbário Central da Universidade Federal de Mato Grosso.

A avaliação qualitativa da composição arbórea foi realizada baseando-se nos parâmetros citados por Milano (1988), Lima (1993) e Albrecht (1998), sendo registradas as seguintes informações:

- Altura total das árvores $(H)$ : mensurada com clinômetro e classificada em pequenas $(\mathrm{H} \leq 6 \mathrm{~m})$, médias $(6,1 \mathrm{~m} \leq \mathrm{H} \leq 10 \mathrm{~m})$ e grandes $(\mathrm{H}>10 \mathrm{~m})$.

- Qualidade: refere-se à condição geral da árvore, considerando o desenvolvimento da copa, caule e raiz, baseando-se para classificação em escalas numéricas conforme apresentado no Quadro 1.

Quadro 1. Escala de classificação da qualidade da copa, caule e raiz das árvores avaliadas*.

\begin{tabular}{|c|c|c|}
\hline $\begin{array}{c}\text { Parte } \\
\text { vegetativa }\end{array}$ & Nota/Atributo & Descrição \\
\hline \multirow{3}{*}{ Copa } & 1 & $\begin{array}{l}\text { Copa ruim, apresentando, galhos quebrados, sinais visíveis de } \\
\text { patógenos ou pragas, principalmente cupins ou características } \\
\text { atípicas da espécie }\end{array}$ \\
\hline & 2 & $\begin{array}{l}\text { Copa razoável, apresentando condições e vigor para o local, ou } \\
\text { seja, danos físicos, pragas ou patógenos com menos } \\
\text { severidade. }\end{array}$ \\
\hline & 3 & $\begin{array}{l}\text { Copa em situação boa, bastante vigorosa e que apresenta a } \\
\text { forma típica da espécie. }\end{array}$ \\
\hline \multirow[t]{2}{*}{ Caule } & 1 & $\begin{array}{l}\text { Anormal, indivíduos que apresentam o caule com formações } \\
\text { atípicas da espécie, por fatores como: condições de } \\
\text { luminosidade, folhagem assimétrica pendendo mais para um } \\
\text { lado, cipós ou declividade do terreno }\end{array}$ \\
\hline & 2 & $\begin{array}{l}\text { Normal, indivíduos que apresentam características típicas da } \\
\text { espécie. }\end{array}$ \\
\hline \multirow{3}{*}{ Raiz } & 1 & $\begin{array}{l}\text { Raízes expostas totalmente desprotegidas, devido a processos } \\
\text { erosivos. }\end{array}$ \\
\hline & 2 & Raízes pouco expostas. \\
\hline & 3 & Raízes totalmente subterrâneas. \\
\hline
\end{tabular}

* Adaptado de Albretch (1998).

- Necessidade de manejo: foi avaliada para cada indivíduo, a necessidade ou não das seguintes práticas: Poda leve (apenas para retirada de ramos que prejudicam a conformação natural das árvores), Poda pesada (poda para a retirada de boa parte da copa, devido ocasionar algum tipo de dano a outras espécies ou usuários do parque), controle fitossanitário (presença de pragas ou doenças visíveis por meio de danificações nas diversas partes das árvores) e remoção do vegetal (indivíduos mortos ou com sintomas irrecuperáveis).

Além das características descritas acima foram observadas informações quanto a origem da espécie (nativa ou exótica). 


\section{RESULTADOS E DISCUSSÕES}

O Parque Antônio Pires de Campos possui 1502 indivíduos arbóreos com cap acima de $15 \mathrm{~cm}$, distribuídos em 50 espécies, pertencentes a 26 famílias, mostrando assim a existência de uma satisfatória diversidade vegetal (Quadro 2).

Quadro 2. Distribuição quantitativa de cada espécie encontrada na arborização do Parque Antônio Pires de Campos, segundo a família, nome científico e vulgar, origem, total de indivíduos e freqüência.

\begin{tabular}{|c|c|c|c|c|c|}
\hline Família & Nome científico & Nome vulgar & Origem & $\mathrm{N}$ Ind & $\begin{array}{c}\text { Freq } \\
(\%)\end{array}$ \\
\hline \multirow{4}{*}{ Anacardeaceae } & Anacardium microcarpum Ducke & Cajueiro & Nativa & 4 & 0,27 \\
\hline & $\begin{array}{l}\text { Astronium fraxinifolium Schott ex } \\
\text { Spreng. }\end{array}$ & Gonçaleiro & Nativa & 436 & 29,03 \\
\hline & Mangifera indica $\mathrm{L}$. & Mangueira & Exótica & 13 & 0,87 \\
\hline & Spondias lutea $\mathrm{L}$. & Cajazinh & Nativa & 7 & 0,47 \\
\hline \multirow[b]{2}{*}{ Annonaceae } & Annona coriacea Mart. & Ata-do-mato & Nativa & 3 & 0,20 \\
\hline & Xylopia aromatica (Lam.) Mart. & $\begin{array}{l}\text { Banana-de- } \\
\text { macaco }\end{array}$ & Nativa & 16 & 1,07 \\
\hline Apocy & Aspidosperma australe Müll. Arg. & Guatambu & Nativa & 8 & 0,53 \\
\hline Arecaceae & $\begin{array}{l}\text { Acrocomia aculeata (Jacq.) Lodd. ex } \\
\text { Mart. }\end{array}$ & Bocaiuveira & Nativa & 30 & 2,00 \\
\hline \multirow{6}{*}{ Bignoneaceae } & Jacaranda mimosifolia D. Don & Jacarandá & Nativa & 49 & 3,26 \\
\hline & Tabebuia ochracea (Cham.) Standl. & Ipê-amarelo & Nativa & 5 & 0,33 \\
\hline & Cybistax antisyphilitica (Mart.) Mart & Pé-de-anta & Nativa & 13 & 0,87 \\
\hline & \multirow{3}{*}{$\begin{array}{l}\text { Jacaranda caroba (Vell.) A. DC. } \\
\text { Tabebuia impitiginosa (Mart. Ex DC.) } \\
\text { Standl. } \\
\text { Tabebuia heptaphylla (Vell.) Toledo }\end{array}$} & Caroba & Nativa & 2 & 0,13 \\
\hline & & Ipê-roxo & Nativa & 9 & 0,60 \\
\hline & & Piúva & Nativa & 27 & 1,80 \\
\hline Bombacaceae & Chorisia speciosa A. St.-Hil. & Paineira & Nativa & 25 & 1,66 \\
\hline Boraginaceae & Cordia glabrata (Mart.) A. DC. & Louro & Nativa & 110 & 7,32 \\
\hline Cecropiaceae & Cecropia pochystochya Trécul & Embaúba & Nativa & 37 & 2,46 \\
\hline Clusiaceae & Kielmeyera coriaceae Mart \& Zucc. & Pau-santo & Nativa & 14 & 0,93 \\
\hline eae & leprosum & $\begin{array}{l}\text { Carne-de- } \\
\text { vaca }\end{array}$ & Nativa & 16 & 1,07 \\
\hline Dilleniaceae & Curatella americana $\mathrm{L}$. & Lixeira & Nativa & 12 & 0,80 \\
\hline \multirow{13}{*}{ Fabaceae } & Albizia hassleri (Chodat) Burkart & Farinheira & Exótica & 135 & 8,99 \\
\hline & $\begin{array}{l}\text { Anadenanthera colubrina (Vell.) } \\
\text { Brenan }\end{array}$ & $\begin{array}{l}\text { Angico- } \\
\text { branco }\end{array}$ & Nativa & 9 & 0,6 \\
\hline & Bauhinia nitida Benth. & Pata-de-vaca & Nativa & 5 & 0,33 \\
\hline & Delonix regia (Bojer ex Hook.) Raf. & Flamboyant & Exótica & 4 & 0,27 \\
\hline & Dimorphandra mollis Benth. & Barbatimão & Nativa & 5 & 0,33 \\
\hline & Dipteryx alata Vogel & Cumbarú & Nativa & 24 & 1,6 \\
\hline & Diptychandra aurantiaca Tul. & $\begin{array}{l}\text { Carvão- } \\
\text { vermelho }\end{array}$ & Nativa & 42 & 2,8 \\
\hline & $\begin{array}{l}\text { Enterolobium contortisiliquum (Vell.) } \\
\text { Morong }\end{array}$ & Chimbúva & Nativa & 18 & 1,2 \\
\hline & $\begin{array}{l}\text { Hymenaea stigonocarpa Mart. Ex } \\
\text { Hayne }\end{array}$ & Jatobá & Nativa & 34 & 2,26 \\
\hline & Leucaena leucocephala (Lam.) de Wit & Leucena & Exótica & 3 & 0,2 \\
\hline & Machaerium acutifolium Vogel & Espinheiro & Nativa & 36 & 2,4 \\
\hline & Peltophorum dubium (Spreng.) Taub. & Faveiro & Nativa & 12 & 0,8 \\
\hline & Pithecolobium inopinathum Ducke & Sete-cascas & Nativa & 4 & 0,27 \\
\hline
\end{tabular}


Quadro 2. Continuação

Continua...

\begin{tabular}{|c|c|c|c|c|c|}
\hline Família & Nome científico & Nome vulgar & Origem & $\mathrm{N}$ Ind & $\begin{array}{c}\text { Freq } \\
(\%)\end{array}$ \\
\hline Flacourtiaceae & Casearia sylvestris L. & Chá-de-frade & Nativa & 53 & 3,53 \\
\hline Lythraceae & Physocalymma scaberrimum Pohl & Aricá & Nativa & 8 & 0,53 \\
\hline Meliaceae & Cedrela fissilis Vell. & Cedro-rosa & Nativa & 6 & 0,40 \\
\hline Myrtaceae & Campomanesia xanthocarpa O. Berg & Gabirobeira & Nativa & 7 & 0,47 \\
\hline Ochnaceae & Ouratea castaneifolia & $\begin{array}{l}\text { Folha-de- } \\
\text { serra }\end{array}$ & Nativa & 11 & 0,73 \\
\hline Opiliaceae & $\begin{array}{l}\text { Agonandra braziliensis Mier ex Benth. } \\
\text { \& Hook.f. }\end{array}$ & Quina-doce & Nativa & 10 & 0,67 \\
\hline Polygonaceae & Triplaris americana $\mathrm{L}$ & Novateiro & Nativa & 8 & 0,53 \\
\hline \multirow[b]{2}{*}{ Rubiaceae } & \multirow{2}{*}{$\begin{array}{l}\text { Genipa americana L. } \\
\text { Tocoyena formosa (Cham. \& Schltdl.) } \\
\text { K. Schum. }\end{array}$} & \multirow{2}{*}{$\begin{array}{l}\text { Jenipapo } \\
\text { Marmelada- } \\
\text { preta }\end{array}$} & Nativa & 37 & 2,46 \\
\hline & & & Nativa & 8 & 0,53 \\
\hline \multirow{2}{*}{ Sapindaceae } & Dilodendron bipinnatum Radlk. & Mulher-pobre & Nativa & 3 & 0,20 \\
\hline & Talisia esculenta (A. St.-Hil.) Radlk. & Pitombeira & Nativa & 58 & 3,86 \\
\hline Simaroubaceae & Simarouba versicolor A.St.-Hil. & Pau-de-perdiz & Nativa & 26 & 1,73 \\
\hline Sterculiaceae & Guazuma ulmifolia Lam. & Chico-magro & Nativa & 27 & 1,80 \\
\hline Tiliaceae & Luehea paniculata Mart. & Açoita-cavalo & Nativa & 2 & 0,13 \\
\hline Verbenaceae & Vitex polygama Cham. & Tarumã & Nativa & 8 & 0,53 \\
\hline \multirow{2}{*}{ Vockysiaceae } & Qualea grandiflora Mart. & Pau-terra & Nativa & 32 & 2,13 \\
\hline & Vochysia haenkeana Mart. & Cambará-liso & Nativa & 4 & 0,27 \\
\hline & & Mortas & & 27 & 1,80 \\
\hline & & & & 1502 & 100,00 \\
\hline
\end{tabular}

Das árvores medidas, as espécies que mais se destacaram em número de indivíduos ao longo de toda área foram: o gonçaleiro (Astronium fraxinifolium), a farinheira (Albizia hasslerii) e o louro (Cordia glabrata) representando $46,32 \%$ do total de indivíduos presentes na área.

As famílias com maior número de espécies (Figura 1) foram: Anacardeaceae $(30,6 \%)$, Leguminosae - Mimosoideae $(11,1 \%)$, Boraginaceae $(7,3 \%)$ e Leguminosae Caesalpinioideae $(5,4 \%)$. Estas famílias representam $54,4 \%$ do total de indivíduos arbóreos ocorrentes, indicando uma maior contribuição destas famílias na área.

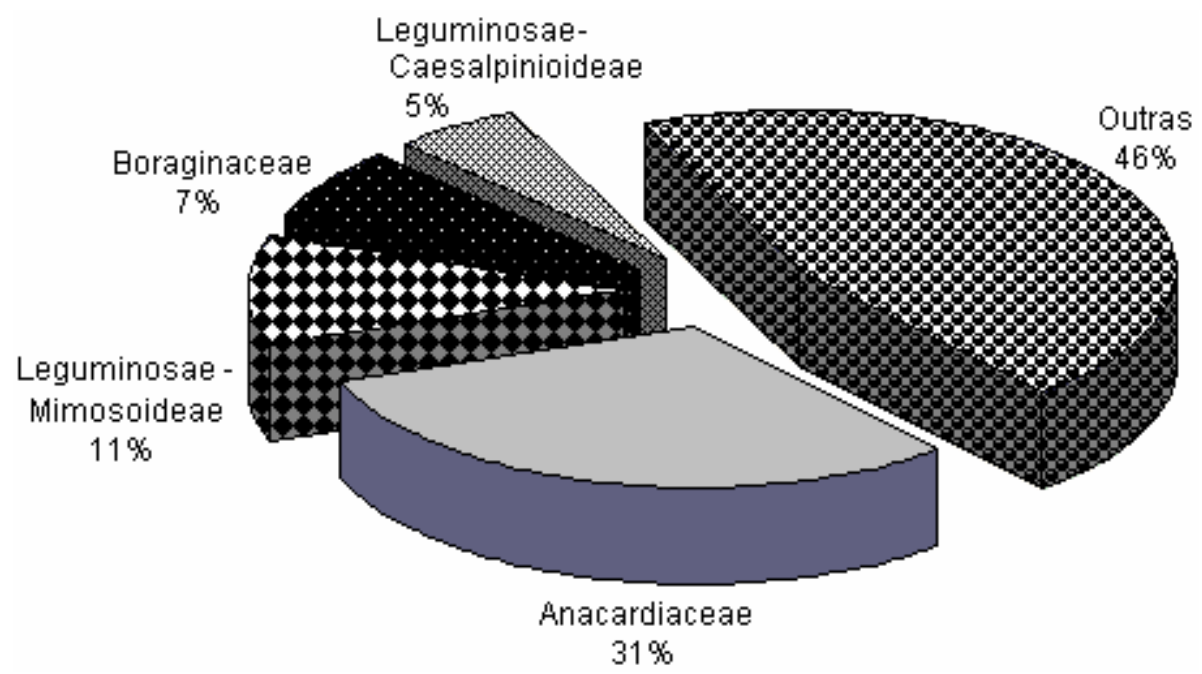


Figura 1. Distribuição dos indivíduos medidos dentro das principais famílias, para a área em estudo.

Observa-se que, a família Anacardeaceae se destaca devido a grande quantidade de indivíduos da espécie Astronium fraxinifolium, que representa cerca de $29,03 \%$ do total de indivíduos, ultrapassando as recomendações de Santamour Júnior (2002) que relata que a maior diversidade de espécies de árvores na paisagem urbana se faz necessária justamente para garantir o máximo de proteção contra pragas e doenças. Dessa forma, segundo o mesmo autor, recomenda-se não exceder mais que 10\% da mesma espécie, $20 \%$ de algum gênero e 30\% de uma família botânica. O Parque Antônio Pires de Campos apresenta-se com apenas uma espécie que ultrapassa os $10 \%$ desejáveis, o gonçaleiro (Astronium fraxinifolium); esse excedente, entretanto, não desqualifica o indivíduo arbóreo, na medida em que são reconhecidas suas funções ambientais. Quanto ao gênero, não há nenhum com distribuição maior que $20 \%$ da arborização da área.

Em relação às famílias ocorrentes no Parque, verificou-se que a distribuição das mesmas é satisfatória conforme as recomendações de Santamour Júnior (2002).

Verificou-se, que $92 \%$ das espécies existentes no Parque são nativas e um percentual de apenas 8\% são exóticas. De acordo com Sanchotene (1985), o uso de nativas regionais, além de visar à preservação das espécies, ainda condiciona um equilíbrio fisiográfico maior, pois incorporam a paisagem urbana, elementos que propiciam sua integração com a paisagem da região.

O enriquecimento das áreas verdes urbanas com espécies nativas de caráter paisagístico representa um ganho em nível de valorização podendo contribuir, em parte, para a conservação das espécies, embora, possam sofrer diferenciações. Essas espécies devem apresentar resistência aos fatores de estresse do meio urbano, adaptabilidade climática, sobrevivência e desenvolvimento no local de plantio, além de resistência ao ataque de pragas e doenças.

\section{Qualificação das espécies}

\section{Porte das árvores}

$\mathrm{Na}$ ausência de informações confiáveis sobre a idade das árvores, ou época de plantio, a avaliação do porte das árvores foi feita para possibilitar as inferências sobre a sua composição etária.

Após a avaliação total da área, observou-se que $55,66 \%$ dos indivíduos são considerados de grande porte (altura acima de 10 metros), 16,31\% possuem altura média $(6,1$ a $10 \mathrm{~m})$ e $28,03 \%$ das árvores são consideradas de baixa estatura. 


\section{Qualidade (copa, raiz e caule)}

O sistema de classificação proposto para avaliar as condições das copas das árvores, numa escala de 1 a 3 mostrou, que 60,12\% das árvores do parque se enquadram na classe 3, ou seja, possuem copas em condições boas, (33,21\%) para classe 2, apresentaram condições e vigor para o local, ou seja, danos físicos, pragas ou patógenos com menos severidade e $6,67 \%$ para classe 1 (copa ruim).

Quanto à raiz constatou-se que $95,18 \%$ dos indivíduos apresentaram raízes profundas (classe 3), 3,02\% para raízes levemente expostas e apenas 1,8\% raízes consideradas desprotegidas (classe 1). Observou-se que mesmo com a declividade do terreno e a falta de cobertura vegetal rasteira, a maioria das árvores apresentaram suas raízes em bom estado de desenvolvimento.

Para avaliação do caule, utilizou-se o código 1 e 2, respectivamente anormais (tortuosidade inerente à espécie) e normais, dessa maneira, a composição arbórea do Parque mostrou os seguintes resultados com relação à qualidade do caule: $35,21 \%$ das árvores estudadas pertencem à classe 1 (tortuosos) e 64,79\% dos indivíduos possuem o caule considerado normal. Atribui-se a tortuosidade à competição por nutrientes, luminosidade e até mesmo por espaço.

\section{Necessidade de manejo}

De acordo com o inventário qualitativo realizado na área foi possível constatar que $19,42 \%$ e $2,63 \%$ do total das árvores necessitam de poda leve e pesada respectivamente, lembrando que a poda deve ser executada na época de repouso vegetativo das árvores. Se os cortes não forem intensos, pode ser realizada em qualquer época do ano. As espécies de folhas perenes devem ser podadas no período que vai do término da frutificação à emissão de novos ramos. As espécies de folhas caducas devem ser podadas após perderem as folhas no fim da fase de repouso vegetativo, que pode ou não coincidir com o inverno.

De modo geral, as árvores urbanas por estarem num habitat artificializado, estão mais sujeitas a incidência de pragas e doenças. Sendo assim, em relação à composição arbórea do Parque verificou-se que $34,82 \%$ dos indivíduos estão infestados por cupins, sendo a farinheira (Albizia hasslerii), pitombeira (Talisia esculenta) e jatobá (Hymenaea stigonocarpa) as espécies com maiores incidências.

Notou-se, também, que em $24,03 \%$ dos indivíduos, havia a presença de hemiparasitas (erva-de-passarinho) em seus ramos. Em levantamento da arborização da cidade de Porto Alegre-RS, $8,62 \%$ dos indivíduos mostravam-se infestados e este percentual foi considerado relativamente baixo (OLIVEIRA e KAPPEL, 1994).

Alguns tipos de danos presentes nos vegetais iniciam-se com podas mal executadas, deixando exposta a área de corte, foco de entrada e desenvolvimento de 
pragas e doença. Com o tempo, essas doenças se alastram para outras partes da árvore, levando-as às vezes, a morte prematura.

Sendo assim, o índice de árvores mortas em pé, encontradas em toda extensão do Parque foi de $1,79 \%$ do total de indivíduos catalogados, podendo cair a qualquer momento, pela baixa resistência às intempéries naturais. Diante dessa realidade, cerca de $82,69 \%$ do total de indivíduos necessitam de alguma prática de manejo.

\section{CONCLUSÕES}

A arborização do Parque Antônio Pires de Campos possui uma satisfatória distribuição de gêneros e famílias, além de possuir uma adequada diversidade de espécies sendo que as mesmas apresentam, no geral, condições satisfatórias de sanidade.

\section{REFERÊNCIAS BIBLIOGRÁFICAS}

ALBRECHT, J.M.F. Análise funcional, composição arbórea e manejo da malha viária das áreas verdes da cidade de São Carlos - SP. 1998. 217f. Tese (Doutorado em Ecologia e Recursos Naturais) - Universidade Federal de São Carlos, São Carlos-SP.

APG II. An update of the Angiosperm Phylogeny Group classification for the orders and families of flowering plants: APG II. Botanical Journal of the Linnean Society, v. 141, n. 4, p. 399-436, 2003.

LIMA, R. F. Principais problemas fitossanitários das plantas ornamentais da cidade do Recife. In: ENCONTRO NACIONAL SOBRE ARBORIZAÇÃO URBANA, 3., 1993, Curitiba, 1993. Anais... Curitiba: SBAU, 1993. p. 124-127.

MENESES, C. H. S. G.; SOUSA, E. B. M.; MEDEIROS, F. P. M.; MENEZES, I. R.; ALBUQUERQUE, H. N.; SANTOS, L. Análise da arborização dos bairros do Mirante e Vila Cabral na cidade de Campina Grande - PB. Revista de Biologia e Ciências da Terra, v. 3, n. 2, p. 1-6, 2003.

MILANO, M. S. Avaliação quali-quantitativa e manejo da arborização urbana de Maringá-PR. 1988. 120f. Tese (Doutorado em Engenharia Florestal) - Universidade Federal do Paraná, Curitiba-PR.

OLIVEIRA, F. B.; KAPPEL, R. B. Incidência de erva-de-passarinho na arborização de ruas de Porto Alegre. In: CONGRESSO BRASILEIRO DE ARBORIZAÇÃO URBANA, 3., 1994, São Luís, MA. Anais... São Luís, MA: SBAU, 1994. p. 335-346.

PAIVA, H. N.; GONÇALVES, W. Florestas urbanas: planejamento para melhoria da qualidade de vida. Viçosa: Aprenda Fácil, 2002. 177p.

SANCHOTENE, M. M. C. Frutíferas nativas úteis à fauna na arborização urbana. Porto Alegre: Feplam, 1985. 311p.

SANTAMOUR JUNIOR, F. S. Trees for urban planting: diversity, uniformity, and common sense. In: METRIA CONFERENCE, 7., 1990, Lisle. Proceedings... Lisle: 1990. p. 57-66. 